\title{
One-step preparation of water-soluble single-walled carbon
}

\section{nanotubes}

\author{
Li Zhang ${ }^{\mathrm{a}}$, Qing-Qing Ni ${ }^{\mathrm{a}, \mathrm{b}^{*}}$, Yaqin $\mathrm{Fu}^{\mathrm{b}}$, Toshiaki Natsuki ${ }^{\mathrm{a}}$ \\ ${ }^{a}$ Faculty of Textile Science \& Technology, Shinshu University, Japan \\ ${ }^{\mathrm{b}}$ Key Laboratory of Advanced Textile Materials and Manufacturing Technology \\ Ministry of Education, Zhejiang Sci-Tech University, Hangzhou 310018, P.R. China
}

\begin{abstract}
A novel one-step process using potassium persulfate (KPS) as oxidant is proposed in this paper to prepare water-soluble single-walled carbon nanotubes (SWNTs). The process without the need for organic solvents and acids is a low-cost, eco-friendly, facile method. Morphology observation by atomic force microscopy (AFM) indicates that the KPS-treated SWNTs were effectively debunded without obvious shortening in their length. The functional groups and thermal stability of the treated SWNTs were analyzed by Raman spectroscopy, X-ray photoelectron spectroscopy (XPS) and thermogravimetric analysis (TGA). XPS results show that several functional groups such as potassium carboxylate $(-\mathrm{COOK})$, carbonyl $(-\mathrm{C}=\mathrm{O})$ and hydroxyl $(-\mathrm{C}-\mathrm{OH})$ groups were formed on the surfaces of the SWNTs, while the TGA results reveal that the quantity of the functional groups can reach to approximately $20 \%$.
\end{abstract}

Keyword: carbon nanotubes; water-soluble; potassium persulfate

\section{Introduction}

Carbon nanotubes (CNTs) exhibit impressive electrical, thermal and mechanical properties, which make them attractive candidates for a variety of potential applications such as biosensors [1,2], field emission devices [3] as well as reinforcement for composites [4,5]. Unfortunately, CNTs have a tendency to aggregate together into bundles, which makes the handling and processing very problematic. Untreated CNTs

\footnotetext{
* Corresponding author. Tel/Fax: +81268 215438.

E-mail address: niqq@shinshu-u.ac.jp (Qing-Qing Ni)

Postal address: Dept. of Functional Machinery \& Mechanics, Shinshu University, 3-15-1 Tokida, Ueda, Japan 386-8567
} 
are normally not water-soluble, but in many cases CNTs are used in their water-soluble form. Therefore, it is extremely important to develop treating processes to prepare dispersed and water-soluble CNTs.

Up to now, many efforts have been made to prepare water-soluble CNTs [6-20] and numerous methods for chemical functionalization of carbon nanotubes, either at the tip or sidewall of CNTs, have already been reported. Among them, functional groups on nanotubes are commonly made by treating them in strong oxidants such as sulfuric acid $\left(\mathrm{H}_{2} \mathrm{SO}_{4}\right)$ and nitric acid $\left(\mathrm{HNO}_{3}\right)$ [18-20]. Gao et al. [18] functionalized and cut carbon nanotubes in concentrated nitric acid and sulfuric acid (volume ration $=1: 3$ ) with constant stirring for $1 \mathrm{~h}$ followed by sonication for $20 \mathrm{~h}$, and the shortened CNTs are well-separated and kept individual from each other. Tchoul et al. [19] used nitric acid to modify CNTs and discussed the effect on dispersibility, size and structure of nanotubes. Osorio et al. [20] functionalized his materials by three different procedures $\left(\mathrm{H}_{2} \mathrm{SO}_{4} / \mathrm{HNO}_{3} / \mathrm{HCl}, \mathrm{H}_{2} \mathrm{SO}_{4} / \mathrm{HNO}_{3}\right.$ and $\left.\mathrm{HNO}_{3}\right)$ and studied the differences and efficiency obtained on these methods, as well as its effect on the dispersion of CNTs in aqueous media. According to the above literatures, the improvement of water-solubility of nanotubes results from inserting carboxylic acid groups $(-\mathrm{COOH})$ on the nanotube surfaces. However, these processes usually accompanied by cutting process and deeply etching, while carboxylic acid groups are difficult to ionize in common aqueous media, so it is very limited to improve the water-solubility.

Herein, we report a facile and effective approach to prepare water-soluble CNTs by the introduction of potassium carboxylate $(-\mathrm{COOK})$ by mild oxidation process using potassium persulfate (KPS). KPS is previously used as a common initiator for emulsion polymerization [21,22], and also an important role as oxidant in organic chemistry [23, 24]. This one-step method without the need for organic solvents and acids may be considered as a low-cost, eco-friendly and convenient method to prepare water-soluble nanotubes. The sketch of the reaction mechanism is showned in Scheme 1. Compare to carboxylic acid groups $(-\mathrm{COOH})$, the potassium carboxylate group (-COOK) on nanotube surfaces is easier to ionize in aqueous media, and the charged functional groups on the surface enable the nanotubes to repel from each other, keeping them uniformly dispersed, therefore the water-solubility is improved obviously. This 
functionalization technique presented here is anticipated to be a fast and effective strategy to prepare water-soluble CNTs and provides an opportunity to modify nanotubes with many other different functional groups.

\section{Experimental}

\subsection{Materials}

Single-walled carbon nanotubes (Wako Pure Chemical Industries Ltd., Japan) were synthesized by arc-discharge method with a diameter of 0.7-2 nm. Potassium persulfate (KPS) and potassium hydroxide $(\mathrm{KOH})$ (Wako Pure Chemical Industries Ltd., Japan) were used without further purification.

\subsection{One-step reaction for preparing water-soluble SWNTs}

$40 \mathrm{mg}$ pristine SWNTs and $50 \mathrm{ml}$ deionized water were added to a flask and dispersed with the aid of an ultrasonic water bath for $60 \mathrm{~min}$ at room temperature. Then $0.45 \mathrm{~g}$ KPS was added to the flask and the $\mathrm{pH}$ value of the reaction system was adjusted to 13 by adding concentrated $\mathrm{KOH}$ solution. The flask equipped with a reflux condenser and a magnetic stir bar was kept at $85{ }^{\circ} \mathrm{C}$ with vigorous mixing for $3 \mathrm{~h}$, and then cooled down to room temperature naturally. The contents of the flask were carefully diluted with water and centrifugated at $3000 \mathrm{rpm}$ for $20 \mathrm{~min}$. The centrifugation process was expected to separate the catalyst and bundled SWNTs from the solution. The supernatant solution was collected and filtered through a hydrophilic polytetrafluoroethylene membrane $(47 \mathrm{~mm} / 0.2 \mu \mathrm{m}$, from Millipore) and washed with distilled water. Finally, the products were dried overnight at $80{ }^{\circ} \mathrm{C}$.

\subsection{Characterization}

The AFM observations were carried out in the dynamic mode using a scanning probe microscope (Shimadzu SPM-9500J3). The sample for AFM analysis was produced by dropping the solution of SWNTs (about $0.01 \mathrm{mg} / \mathrm{mL}$ ) onto a freshly cleaved mica substrate, and drying in the air. Raman spectroscopy measurements were carried out on a HoloLab series 5000 Raman spectroscope (Kaiser optical systems, INC.) furnished with a $514 \mathrm{~nm}$ laser excitation. X-ray photoelectron spectroscopy (XPS) analyses were used to measure the elemental composition of the samples and 
characterize the chemical states of carbon. XPS measurements were performed on a Kratos Axis Ultra DLD x-ray photoelectron spectrometer with a standard MgKa $(1256.6 \mathrm{eV}) \mathrm{x}$-ray source operated at $10 \mathrm{~mA}$ and $15 \mathrm{kV}$. All binding energies were referenced to $\mathrm{Au}(4 \mathrm{f} 7 / 2)$ at $84 \mathrm{eV}$. The thermogravimetrical analysis (TGA) was preformed on a Rigaku Thermo Plus TG8120 apparatus (Rigaku Denki, Japan) under nitrogen atmosphere at $10{ }^{\circ} \mathrm{C} / \mathrm{min}$ in temperature range from $30{ }^{\circ} \mathrm{C}$ to $800{ }^{\circ} \mathrm{C}$.

\section{Results and Discussion}

The KPS-treated SWNTs exhibit obvious improvement of water-solubility, which can be readily observed by visual inspection. Figure 1 shows that while the pristine SWNTs start aggregating rapidly after sonication, the treated nanotubes form stable suspensions without visible aggregation even after one month. The highest SWNTs concentration of stable nanotubes solution is around $1.4 \mathrm{mg} / \mathrm{mL}$ according to literature methods [25]. These indicate that the solubility and stability of treated nanotubes are strongly improved. In order to further support the enhanced dispersion property of treated nanotubes in water, an atomic force microscopy (AFM) analysis of pristine SWNTs and treated SWNTs was carried out. Figure $2 \mathrm{~b}$ shows a representative AFM image of the KPS-treated SWNTs. The mean diameter of the nanotubes (measured at 20 points), equal to the mean top height of nanotubes [26,27], is calculated to be $3.27 \mathrm{~nm}$. It is interesting that we also find some top heights in the range of $0.7-2 \mathrm{~nm}$ which may correspond to the individual dissolved nanotubes. Comparing that the diameter of the pristine nanotubes used in this study is about $11.35 \mathrm{~nm}$ (measured at 20 points, see Fig. 2a), the result indicates that the treatment by KPS can effectively debundle SWNTs Moreover, after KPS treatment and centrifugation process, the nanotubes show no obvious shortening and amorphous nanoparticles attached to the nanotubess reduced markedly.

Raman spectroscopy is a powerful tool to characterize the degree of functionlization of SWNTs. The D/G ratio is the ratio of the disorder mode (D band, $1327 \mathrm{~cm}^{-1}$ ) to the tangential mode ( $\mathrm{G}$ band, $\left.1580 \mathrm{~cm}^{-1}\right)$. It is related to the amount of $\mathrm{sp}^{3}$-hybridized carbon atoms in the SWNTs and thus provides direct information of the degree of sidewall functionalization, in which $\mathrm{sp}^{2}$-hybridized carbons are converted into 
$\mathrm{sp}^{3}$-hybridized carbons [28]. According to Figure 3, the KPS-treated nanotubes have a significant increase in the $\mathrm{D} / \mathrm{G}$ ratio from 0.22 to 0.51 . Previous studies have indicated that the increased $\mathrm{D} / \mathrm{G}$ ratio corresponds to the increased degree of functionalization $[28,29]$. The observed increase of $\mathrm{D} / \mathrm{G}$ ratio here also suggests that the high degree of functionalization of SWNTs is obtained after KPS treatment.

The Raman data strongly support the conclusion that the functionalization did occur, but it could not show direct chemical bonding informations on the nanotube surfaces. For KPS treatment process, there are two possible mechanisms for preparing water-soluble nanotubes. Under alkaline condition, KPS is possible to oxidize pristine nanotubes into water-soluble nanotubes by introducing hydrophilic groups on the nanotube surfaces such as carboxylic anion $\left(-\mathrm{COO}^{-}\right)$. On the other hand, KPS is a common initiator and possible to produce free radicals such as sulfate radical anion $\left(\mathrm{SO}^{-}{ }^{-}\right)$when heated in aqueous environment. If $\mathrm{SO}^{-{ }^{-}}$is attached to the nanotube surfaces, sulfonated salt will be formed on the surface, which probably makes the nanotubes dissolved in water just as reported by Liang et al. [13]. In order to provide direct evidence for the added functional groups on the nanotube surfaces, XPS analysis was performed on both the pristine nanotubes and treated nanotubes. The typical XPS spectra are presented in Figure 4. Survey scans of treated nanotubes (Figure 4a) show the presence of carbon, oxygen and potassium. However, the high resolution $\mathrm{S} 2 \mathrm{~s}$ spectrum (Figure 4d) shows that no sulfur is detected, which reveals no sulfonated salt was formed on the nanotube surfaces during modification. The atomic percentages are 68.50 for $\mathrm{C} 1 \mathrm{~s}, 25.14$ for $\mathrm{O} 1 \mathrm{~s}$ and 6.36 for $\mathrm{K} 2 \mathrm{~s}$, indicating the water-soluble nanotubes most probably result from the formation of $-\mathrm{COOK}$ on the nanotube surfaces.

In order to confirm the existence of $-\mathrm{COOK}$, the high resolution of $\mathrm{C} 1 \mathrm{~s}$ was analyzed with a fitting routine by decomposing $\mathrm{C} 1 \mathrm{~s}$ spectrum into individual mixed Gaussian-Lorentzian peaks after a Shirley background subtraction. After the curve-fitting of the $\mathrm{C} 1 \mathrm{~s}$ spectrum (Figure 5), the overall peak of $\mathrm{C} 1 \mathrm{~s}$ in the region (282-292 eV) could be fitted by a superposition of four peaks (the peaks of $\mathrm{K} 2 \mathrm{p}$ at $293.2 \mathrm{eV}$ and $295.9 \mathrm{eV}$, effecting C 1s spectrum, should be taken into account during curve fitting). The main binding energy peak $(285.0 \mathrm{eV})$ is attributed to the $\mathrm{C}-\mathrm{C} 1 \mathrm{~s}$, while the other three peaks are assigned to $-\mathrm{C}-\mathrm{OH}(286.2 \mathrm{eV}),-\mathrm{C}=\mathrm{O}(287.6 \mathrm{eV})$, and 
-COOK (288.9 eV), respectively [30,31]. According to Figure 5, we can quantitatively calculate each functional group based on the ratios of peak areas. The percentages are $70.8 \%$ for $\mathrm{C}-\mathrm{C} 1 \mathrm{~s}, 12.7 \%$ for $-\mathrm{C}-\mathrm{OH}, 5.0 \%$ for $-\mathrm{C}=\mathrm{O}$, and $11.5 \%$ for $-\mathrm{COOK}$. These values give us rough information about the functional groups attached to nanotubes. However, it is clear to confirm the existence of -COOK. Interestingly, a simple experiment can serve as an auxiliary proof for the existence of $-\mathrm{COOK}$ : when hydrochloric acid $(\mathrm{HCl})$ was added to the KPS-treated nanotubes aqueous solution to adjust the $\mathrm{pH}$ value to about 2 , the solution began to flocculate partially, probably resulting from the transformation from $-\mathrm{COOK}$ to $-\mathrm{COOH}$ and the $-\mathrm{COOH}$ on nanotube surfaces is not so easy to ionize in aqueous solution as - COOK.

The thermogravimetric analyses were studied to examine the thermal stability of the KPS-treated SWNTs and quantify the functional groups on the surface of nanotubes. The results are presented in Figure 6 and, as expected, the thermal degradation of KPS-treated nanotubes shows a multistage process. The first stage, up to a temperature of $200{ }^{\circ} \mathrm{C}$, a weight loss of approximately $4 \%$ corresponds to the evaporation of the absorbed water. The second stage in the range of $200-600{ }^{\circ} \mathrm{C}$ is attributed to the decomposition of functional group on the nanotube surfaces, such as carboxylic group and hydroxyl group. And the mass loss at the temperature above $600{ }^{\circ} \mathrm{C}$ is assigned to the decomposition of nanotubes [32]. According to the TGA trace, the quantity of functional groups on the nanotube surfaces is estimated to be nearly $20 \%$. It is noted that the treated SWNTs undergo a lower temperature of decomposition compared to the pristine SWNTs, and this result confirms the effective functionalization on the nanotube surfaces. The TGA results are in agreements with the Raman and the XPS results, confirming that the specific treatment is a very effective and facile method to functionalize the pristine SWNTs.

\section{Conclusions}

Water-soluble SWNTs (solubility of $1.4 \mathrm{mg} / \mathrm{mL}$ ) were synthesized by one-step oxidation process using potassium persulfate (KPS) as oxidant. The modified SWNTs show greater ease of exfoliation into water than pristine SWNTs, confirmed by AFM 
analysis (mean diameter of $3.27 \mathrm{~nm}$ ). XPS results show that potassium carboxylate $(-\mathrm{COOK})$, carbonyl $(-\mathrm{C}=\mathrm{O})$ and hydroxyl $(-\mathrm{C}-\mathrm{OH})$ groups were formed on nanotube surfaces, and the quantity of the functional groups on the nanotube surfaces was about $20 \%$ calculated from TGA data. The strategy may also be used to functionalize multiwalled carbon nanotubes and other nanostructures. The convenient approach here is expected to be a common approach to achieve water-soluble carbon nanotubes in the further.

\section{Acknowledgments}

This work was partly supported by Grant-in-Aid for Global COE (center of excellent) Program by the Ministry of Education, Culture, Sports, Science, and Technology, Japan. 


\section{References}

[1] P.W. Barone, S. Baik, D.A. Heller, M.S. Strano, Nat. Mater. 4 (2004) 86.

[2] S.N. Kim, J.F. Rusling, F. Papadimitrakopoulos, Adv. Mater. 19 (2007) 3214.

[3] J.M. Bonard, M. Croci, C. Klinke, R. Kurt, O. Noury, N. Weiss, Carbon 40 (2002) 1715.

[4] J.N. Coleman, U. Khan, Y.K. Gun'ko, Adv. Mater. 18 (2006) 689.

[5] J.N. Colemana, U. Khana, W.J. Blaua, Y.K. Gun'ko, Carbon 44 (2006) 1624.

[6] M.J. O'Connell, P. Boul, L.M. Ericson, C. Huffman, Y. Wang, E. Haroz, C. Kuper, J. Tour, K.D. Ausman, R.E. Smalley, Chem. Phys. Lett. 342 (2001) 265.

[7] J. Chen, H. Liu, W.A. Weimer, M.D. Halls, D.H. Waldeck, G.C. Walker, J. Am. Chem. Soc. 124 (2002) 9034.

[8] B. Rajdip, N.R. Einat, R. Oren, Y.R. Rachel, Nano Lett. 2 (2002) 28.

[9] K. Nobusawa, A. Ikeda, J. Kikuchi, S. Kawano, N. Fujita, S. Shinkai, Angew. Chem. Int. Ed. 47 (2008) 4577.

[10] M. Klink, H. Ritter, Macromol. Rapid Commun. 29 (2008) 1208.

[11] V.C. Moore, M.S. Strano, E.H. Haroz, R.H. Hauge, R.E. Smalley, Nano Lett. 3 (2003) 1379.

[12] C. Hu, Z. Chen, A. Shen, X. Shen, J. Li, S. Hu, Carbon 44 (2006) 428.

[13] F. Liang, J.M. Beach, P.K. Rai, W. Guo, R.H. Hauge, M. Pasquali, R.E. Smalley, W. E. Billups, Chem. Mater. 18 (2006) 1520.

[14] J.L. Hudson, H. Jian, A.D. Leonard, J.J. Stephenson, J.M. Tour, Chem. Mater. 18 (2006) 2766.

[15] J.J. Stephenson, J.L. Hudson, S. Azad, J.M. Tour, Chem. Mater.18 (2006) 374.

[16] J. Chattopadhyay, F.J. Cortez, S. Chakraborty, N.K.H. Slater, W.E. Billups, Chem. Mater. 18 (2006) 5864.

[17] R. Tian, X. Wang, M. Li, H. Hu, R. Chen, F. Liu, H. Zheng, L. Wan, Appl. Surf. Sci. 255 (2008) 3294.

[18] B. Jia, L. Gao, J. Phys. Chem. B 111 (2007) 5337.

[19] M.N. Tchoul, W.T. Ford, G. lolli, D.E. Resasco, S. Arepalli, Chem. Mater. 19 (2007) 5765 . 
[20] A.G. Osorio, I.C.L. Silveira, V.L. Bueno, C.P. Bergmann, Appl. Surf. Sci. 255 (2008) 2485.

[21] R.W. Simms, M.D. Hoidas, M.F. Cunningham, Macromolecules 41 (2008) 1076.

[22] W. Bai, L. Zhang, R. Bai, G. Zhang, Macromol. Rapid Commun. 29 (2008) 562.

[23] J. Kronholm, P. Jyske, M.L. Riekkola, Ind. Eng. Chem. Res. 39 (2000) 2207.

[24] Y. Lian, Y. maeda, T. Wakahara, T. Akasaka, S. Kazaoui, N. Minami, T. Shimizu, N. Choj, H. Tokumoto, J. Phys. Chem. B. 108 (2004) 8848.

[25] J. Zou, L. Liu, H. Chen, S.I. Khondaker, R.D. McCullough, Q. Huo, L. Zhai, Adv. Mater. 20 (2008) 2055.

[26] M. Shigeta, M. Komatsu, N. Nakashima, Chem. Phys. Lett. 418 (2006) 115.

[27] M. Okamoto, T. Fujigaya, N. Nakashima, Adv. Func. Mater. 18 (2008) 1776.

[28] R. Graupner, J. Raman Spectrosc.38 (2007) 673.

[29] H. Hayden, Y.K. Gun'ko, T.S. Perova, Chem. Phys. Lett. 435 (2007) 84.

[30] A.M. Puziy, O.I. Poddubnaya, Carbon 36 (1998) 45.

[31] N. Kovtyukhova, E. Buzaneva, A. Senkevich, Carbon 36 (1998) 549.

[32] J.B. Kim, T. Premkumar, K. Lee, K.E. Geckeler, Macromol. Rapid Commun. 28 (2007) 276. 


\section{Figure captions}

Scheme1. Reaction scheme for the treatment of SWNTS using KPS.

Figure 1. Optical photographs of (a) pristine SWNTs and (b) KPS-treated SWNTs in water. (A) Immediately after sonication; (B) after $1 \mathrm{~min}$; (C) after 5 min; (D) after $1 \mathrm{~h}$; (E) after 1 day; and (F) after 30 days.

Figure 2. Typical AFM images of pristine SWNTs and KPS-treated SWNTs solution taken after it was cast and dried on a mica substrate. (a) pristine SWNTs and (b) KPS-treated SWNTs (left), and the top height profiles at four points (right).

Figure 3. Raman spectrum of (a) pristine SWNTs and (b) KPS-treated SWNTs.

Figure 4. XPS spectra of KPS-treated SWNTs. (a) survey of the spectral region from -10 to $1150 \mathrm{eV}$, (b) the narrow scan of carbon 1s region, (c) the narrow scan of the oxygen 1s region, (d) the narrow scan of the potassium 2 s region, and (e) the narrow scan of the sulfur 2 s region.

Figure 5. The curve fitting of the carbon 1s of KPS-treated SWNTs.

Figure 6. TGA curves of (a) pristine SWNTs and (b) KPS-treated SWNTs. 


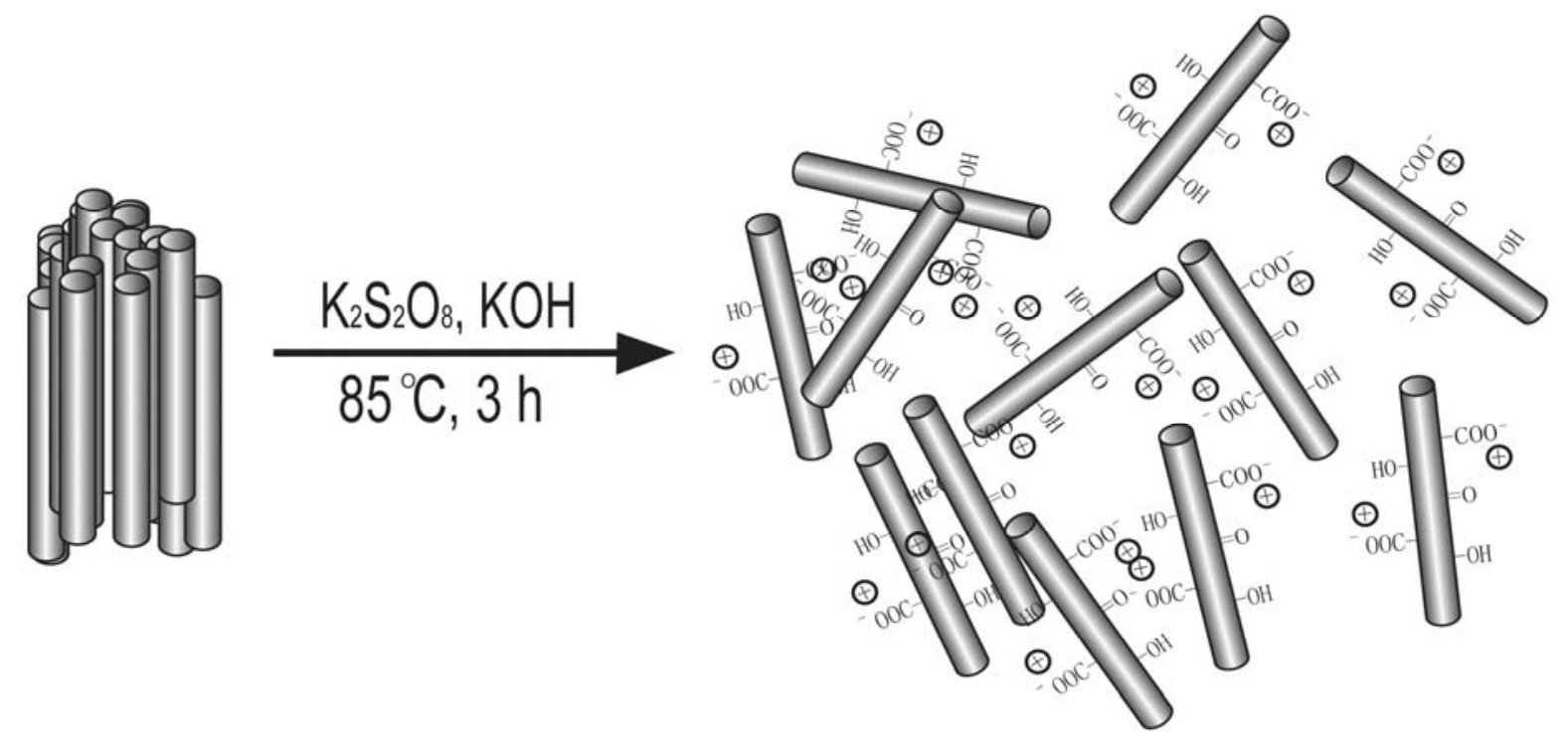

Scheme1. 

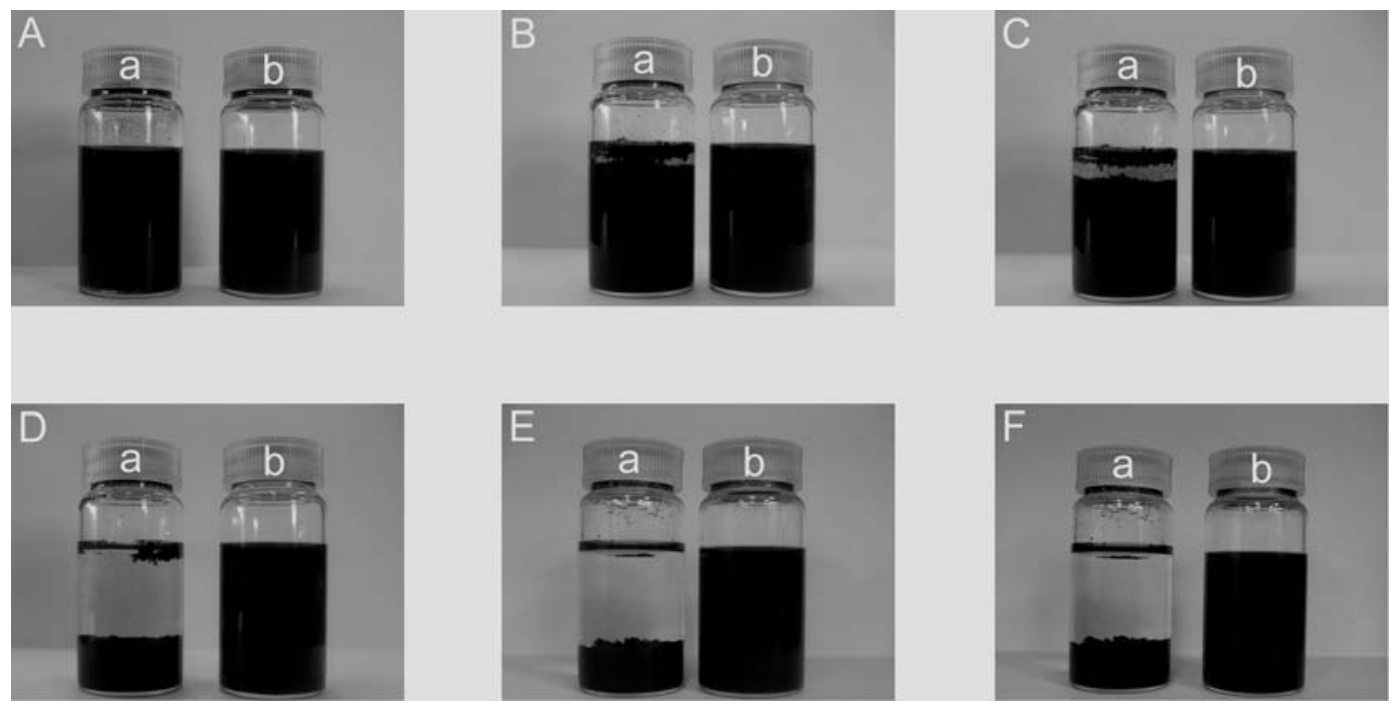

Figure 1. 

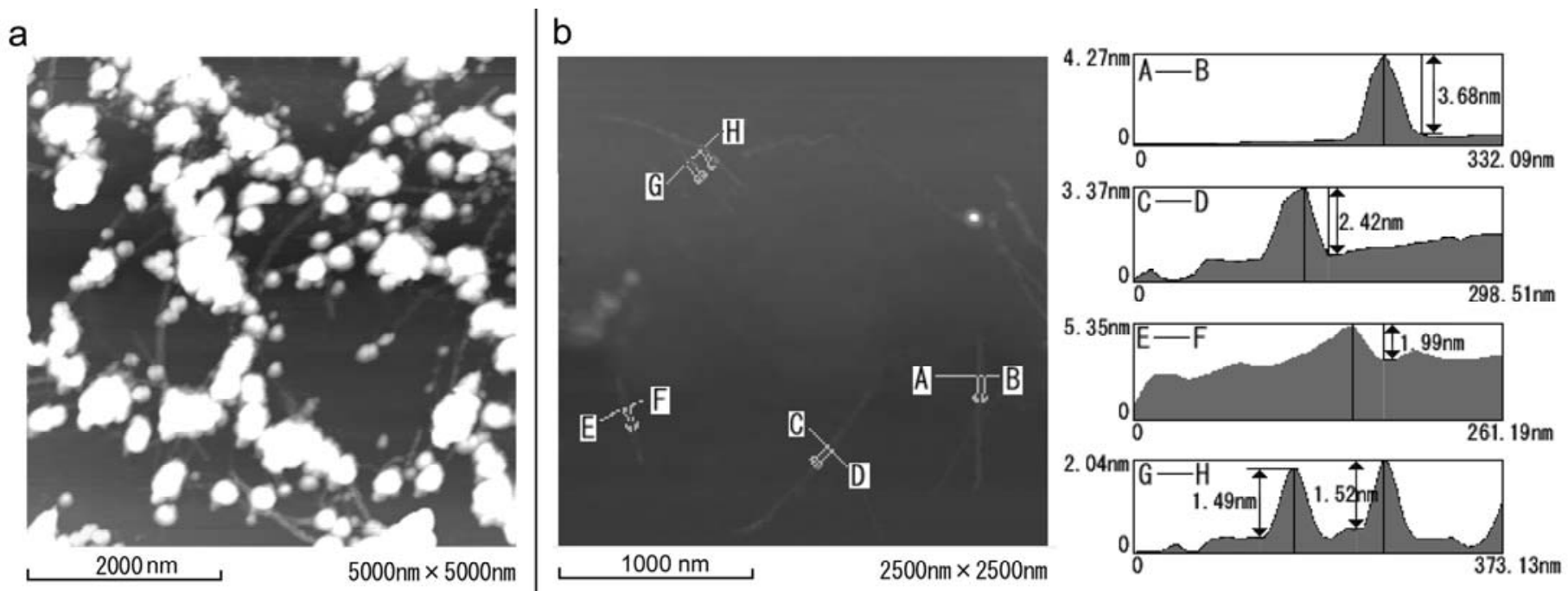

Figure 2. 


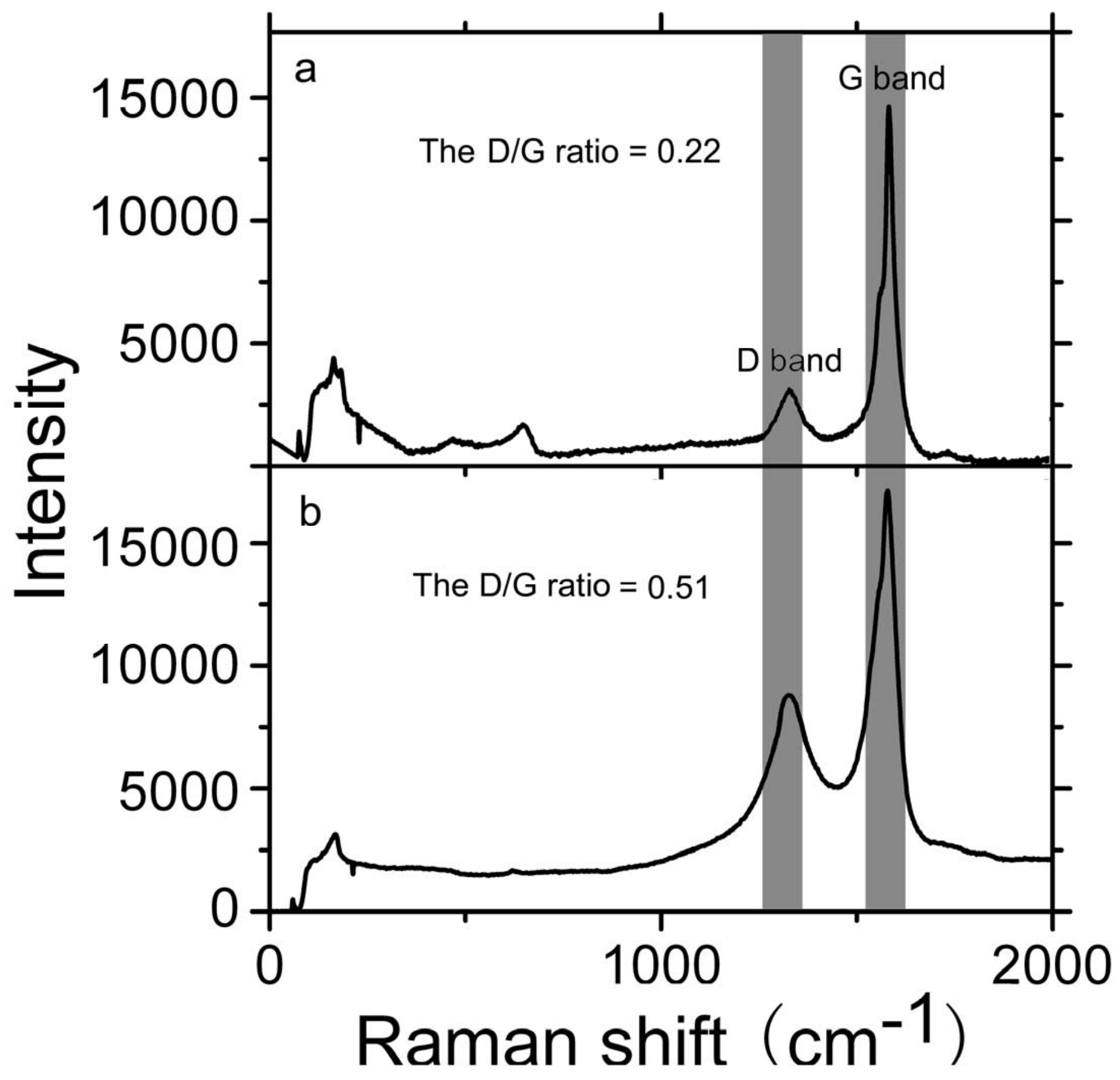

Figure 3. 

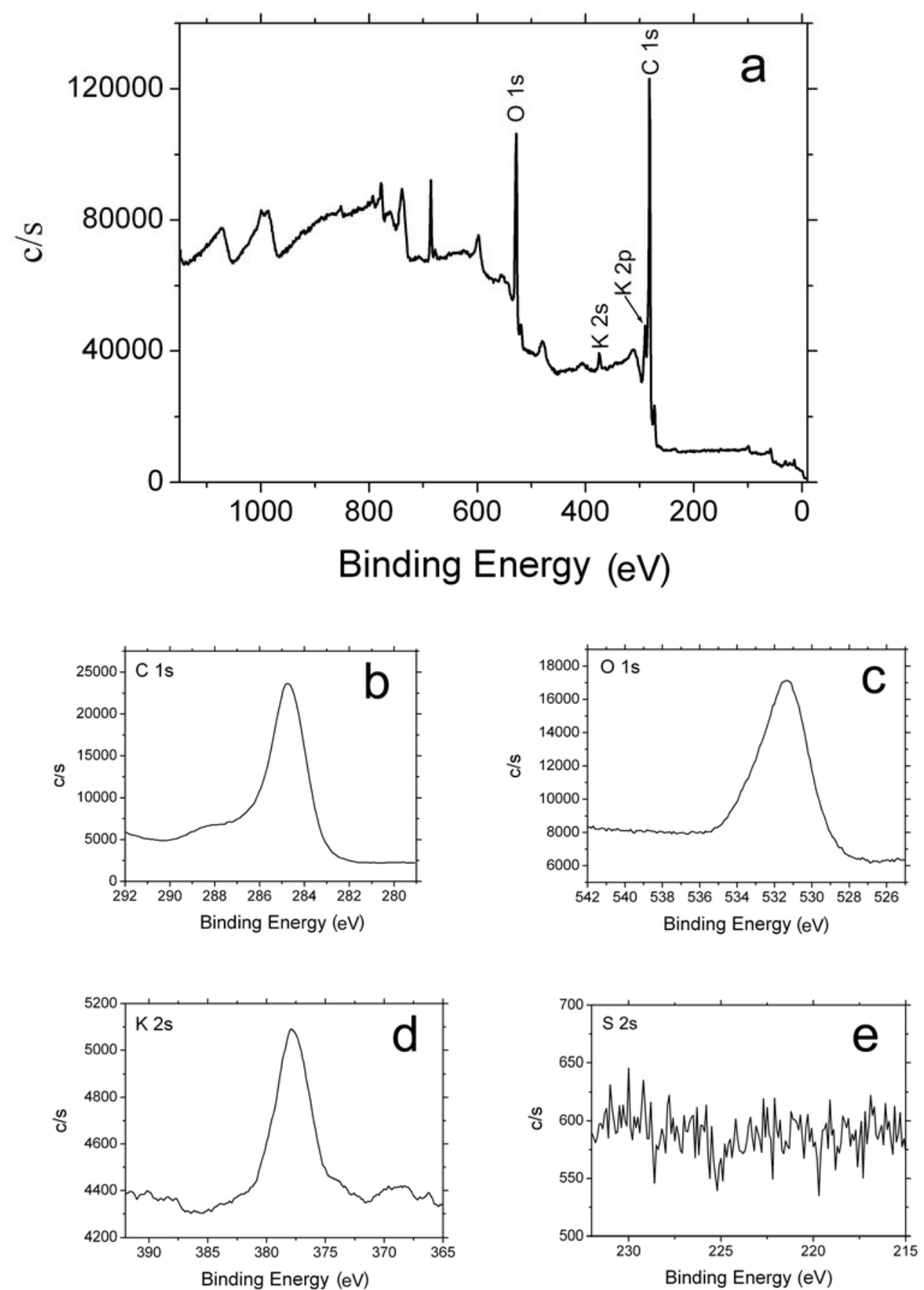

Figure 4. 


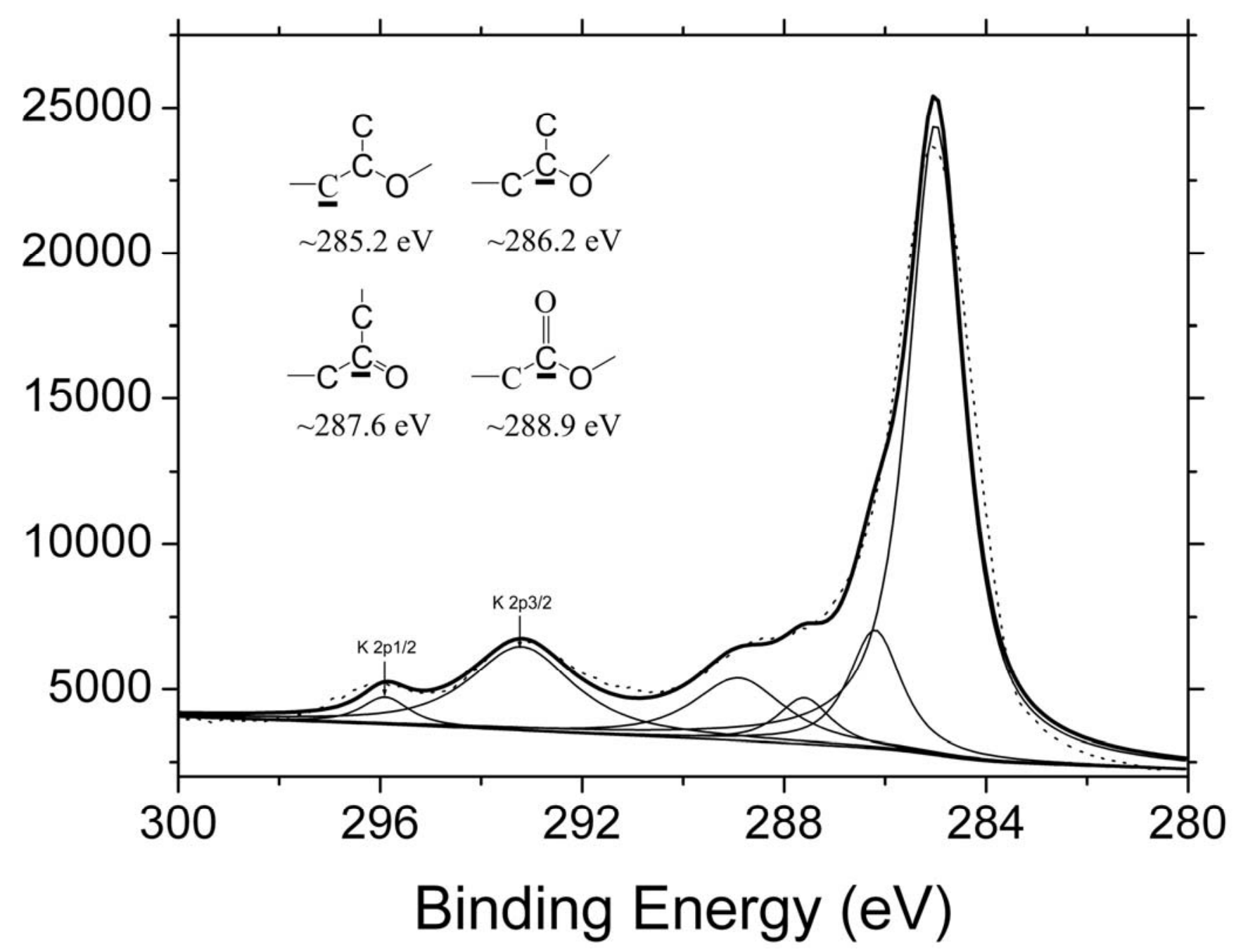

Figure 5. 


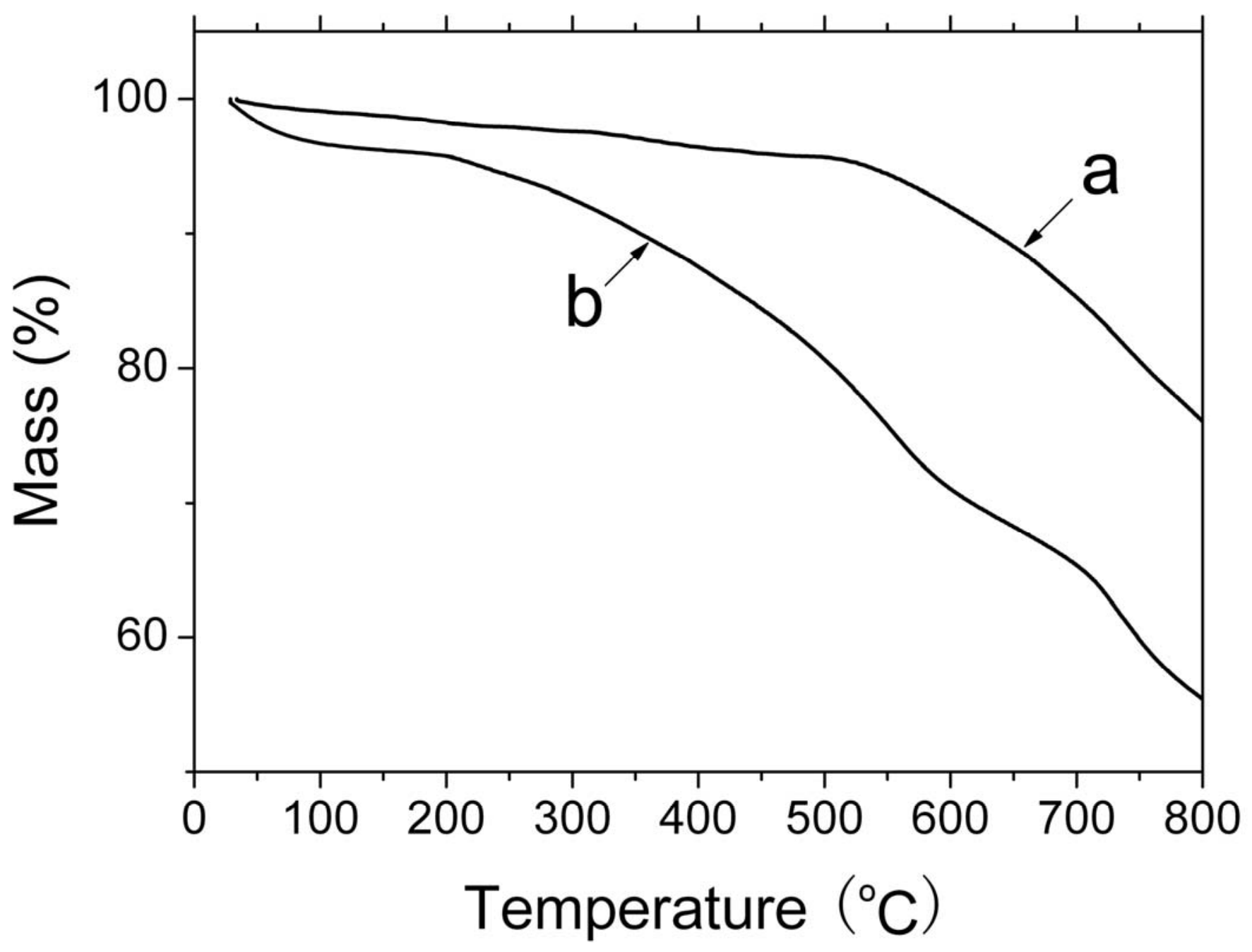

Figure 6. 